\title{
Drug Discovery Enhanced by Artificial Intelligence
}

\author{
Woo Sung Son* \\ College of Pharmacy and Institute of Pharmaceutical Sciences, CHA University, 120 Haeyong-ro, Pocheon-si, Gyeonggi-do, 11160, Republic of Korea
}

Received: 制: October 08, 2018; Published: 制: December 12, 2018

*Corresponding author: Woo Sung Son, College of Pharmacy and Institute of Pharmaceutical Sciences, CHA University, 120 Haeryongro, Pocheon-si, Gyeonggi-do, 11160, Republic of Korea

\section{Abstract}

Artificial intelligence, one of the key technologies in the era of the 4th Industrial Revolution, is expected to greatly affect traditional drug discovery. Artificial intelligence is attracting attention as an innovative technology that can dramatically reduce the high cost and time required for the new drug discovery. At this stage, artificial intelligence is mainly used to search for candidate molecules during drug discovery, but it is highly likely to be actively used in drug discovery through open innovation in the future. In this paper, it is presented the current state of artificial intelligence in each stage of a new drug discovery and prospect for the future usability.

Keywords: Artificial Intelligence; Drug Discovery; Drug Targets; Drug Candidates; Clinical Trials; Post Monitoring

Abbreviations: AI: Artificial Intelligence; SBDD: Structure-Based Drug Discovery; NMR: Nuclear Magnetic Resonance Spectroscopy; CMC: Chemistry, Manufacturing, and Control; GMP: Good Manufacturing Practice; OCR: Optical Character Recognition

\section{Introduction}

The Fourth Industrial Revolution, which has been widely publicized through the Davos Forum in 2016, has had a profound impact on the industry. Especially in the pharmaceutical industry, which requires a lot of resources and time, it is expected that a new breakthrough that maximizes efficiency will be achieved through the 4th Industry Revolution. Artificial intelligence (AI) and big data analysis technologies, which are leading technologies in the 4 th Industrial Revolution era, are expected to have a great impact on the new drug discovery (Figure 1). Many pharmaceutical companies have performed the rational drug discovery through various omics and structure-based drug development. Now they are making great changes through the fusion of $\mathrm{AI}$ and the previously developed technologies for drug discovery [1-3].

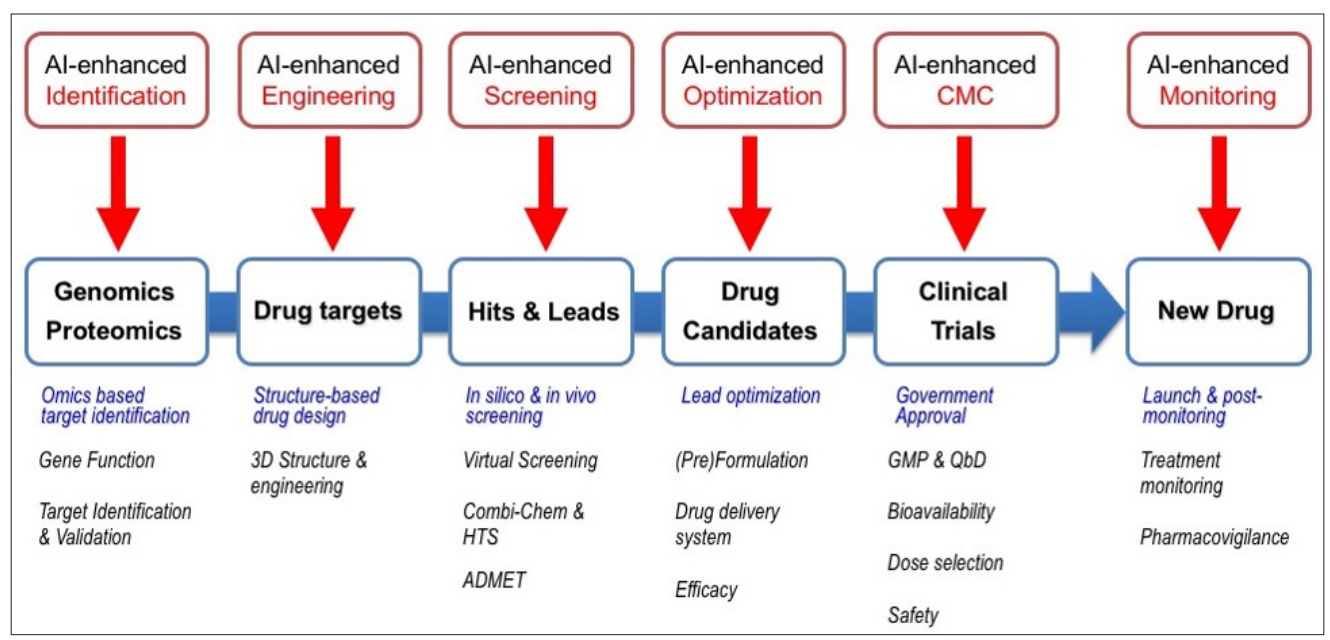

Figure 1: Cycle of rational new drug discovery and application of artificial intelligence. 
The traditional drug discovery is a high-risk, high-return industry that is costly and time-consuming, although it can bring enormous benefit if successful. As of 2016, an average of more than 10 years and $\$ 2.6$ billion are required to develop one new drug [4]. For this reason, in the field of a new drug discovery, pharmaceutical companies are pursuing a strategy to reduce the risk of failure such as open innovation and increase the possibility of success. AI could be attracting attention as a key to dramatically reduce costs and time in the pharmaceutical industry. So far, the AI is mainly used in the early stage of drug discovery and search for candidate compounds in the pharmaceutical industry, but AI will be used for various purposes in order to drastically reduce cost and time for new drug discovery.

\section{AI-Enhanced Identification}

Rational drug discovery, which identify and validates the targets of a new drug discovery based on large amounts of genetic and protein information such as genomics and proteomics, has been the mainstream of pharmaceutical industry. Compared with the conventional screening method based on random screening, it was able to utilize time and resources relatively efficiently. The target selection method based on omics is a crucial process for selecting a valid target from numerous candidates. Therefore, selection and validation of new drug targets can be facilitated with the help of AI. In addition, AI can be actively used to improve the efficiency of drug repurposing, which uses existing drugs already marketed in other diseases, and Watson Drug Discovery developed by IBM have presented the increased efficiency of the drug repurposing using $\mathrm{AI}[5]$.

\section{AI-Enhanced Engineering}

Because the selected targets for a new drug discovery are proteins in most cases, engineering and obtaining threedimensional structures of proteins are important keys to increase efficiency of the entire steps of drug discovery including virtual screening step. Also, those are essential elements of structurebased drug discovery (SBDD) used nowadays in most big pharma, and the three-dimensional structures of proteins are mainly determined using X-ray crystallography and nuclear magnetic resonance spectroscopy (NMR) methods. To develop a new drug based on the protein target, it is necessary to search for active site and validate the target protein for efficacy confirmation. AI could be helpful for selecting mutation site for protein property modification during in vitro and in vivo experiments. In the process of designing or engineering bio drugs including biosimilar, which is growing rapidly in the new drug market, it is a crucial process to transform proteins, so it can be used like application of AI to protein engineering process. For example, Atom wise company actively use $\mathrm{AI}$ in drug design through target engineering as it has developed the first AI technology available for three-dimensional drug design [6].

\section{AI-Enhanced Screening}

Traditionally, searching active compounds is a painful process in drug discovery because it is necessary much time and resources in screening step. Modern rational drug discovery uses molecular docking as a core technique to perform virtual screening and computationally select compounds that are effective among large libraries of compounds. Many docking algorithms have been developed and used for this purpose, but the virtual screening through the in silico method does not yield perfectly hits or lead compounds. Therefore, if the AI method such as machine learning is applied to the existing molecular docking method focusing on the scoring function, the efficiency of screening the active compound can be maximized. Many pharmaceutical companies are focusing on utilizing AI now of virtual screening along with drug repurposing. In addition to structure-based virtual screening, the $\mathrm{AI}$ in the screening step can be used for predicting and verifying the ADMET parameters used in pharmacokinetics and can also affect the efficiency of the drug repurposing mentioned above.

\section{AI-Enhanced Optimization}

AI can also be utilized in the process of optimizing the obtained lead compounds to be commercialized. It is important to develop an effective drug delivery system through a formulation process that optimizes the lead compounds to derive drug candidates. Recently, a variety of material engineering methods have been introduced for the development of a target-oriented drug delivery system. In a typical drug delivery system using an amphipathic substance such as micelle or liposome, a proper combination of drug and other substances is required. To make such a drug delivery system, there are many cases of material composition and environment selection, so that it is possible to shorten the time and resources for drug candidates by preferentially selecting composition ratios and environments that are likely to be successful with the help of AI [7]. In addition, AI can be used to increase the accuracy of toxicity prediction, preclinical experimental design and analysis of experimental results.

\section{AI-Enhanced CMC}

Drugcandidates thatare very close to thenewdrugwilleventually reach clinical trials, the biggest obstacles to drug development, and will require approval of administrative procedures, such as FDA CMC (Chemistry, Manufacturing, and Control), to be licensed as commercial drugs. Along with successful clinical trial results, there is room for AI to be used in the manufacturing and licensing step to be approved as a new drug. Selection of subjects using AI can increase the efficiency and reliability of clinical trials as appropriate screening is the key to successful clinical trials. If AI is used to optimize clinical trial design, the accuracy of clinical outcome analysis can be improved. Although it is still in the early stage, efficiency-oriented production processes that utilize data such as smart factories during GMP (Good Manufacturing Practice)-based drug manufacturing are likely to be necessary for AI utilization.

\section{AI-Enhanced Monitoring}

In the monitoring process after the new drug is released as a commercial product, AI can be used to improve the efficiency of pharmacovigilance. Using AI in the pharmacovigilance step will improve compliance and accelerate customized drug guidance. For instance, GPVAI of Genpact company is currently providing 
solutions for OCR (Optical Character Recognition) and AI to analyze, monitor and control information related to worldwide drug side effects reports.

\section{Conclusion}

New drug discovery is a vast resource and time consuming field, and a wide variety of specialized skills must be integrated in each step. Therefore, in order to increase the efficiency of a new drug discovery, it is necessary to improve the efficiency of all steps during drug discovery, and AI can be very helpful for whole steps in a new drug discovery. So far, AI has been used mainly in the step of searching for candidate molecules in addition to drug repurposing, but it is certain that the necessity of AI will increase in the future. Also, if AI succeeds in improving the accuracy of go / no-go decision support during drug discovery, it is possible to make dramatically improvement of the efficiency of a new drug discovery.

\section{Acknowledgement}

This work is supported in part by grants provided by the Basic Science Research Program through the National Research Foundation of Korea (NRF) funded by the Ministry of Education, Science and Technology (NRF-2017R1D1A1B03030723). This

ISSN: 2574-1241

DOI: _10.26717/BJSTR.2018.12.002189

Woo Sung Son. Biomed J Sci \& Tech Res

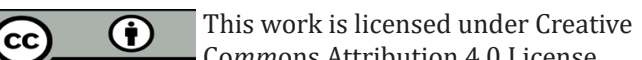

Submission Link: https://biomedres.us/submit-manuscript.php study also partly supported by the GRRC program of Gyeonggi province (GRRC-CHA2017-A01, Discovery and Analysis of Regional Specialized Resources and Operation of Regional Research Service Center).

\section{References}

1. Gawehn E, Hiss JA, Schneider G (2016) Deep Learning in Drug Discovery. Mol Inform 35(1): 3-14.

2. Hessler G, Baringhaus KH (2018) Artificial Intelligence in Drug Design. Molecules 23(10): E2520.

3. Zhavoronkov A (2018) Artificial Intelligence for Drug Discovery, Biomarker Development, and Generation of Novel Chemistry. Mol Pharm 15(10): 4311-4313.

4. Holmes D (2016) A new chapter in innovation. Nature 533(7602): S5455.

5. See HQ, Chan JN, Ling SJ, Gan SC, Leong CO, et al. (2018) Advancing Pharmacy Service using Big Data - Are We Fully Utilising the Big Data's Potential Yet? J Pharm Pharm Sci 21(1): 217-221.

6. Wang C, Xu P, Zhang L, Huang J, Zhu K, et al. (2018) Current Strategies and Applications for Precision Drug Design. Front Pharmacol 9: 787.

7. Sable P, Khanvilkar V (2018) Pharmaceutical Applications of Artificial Intelligence. Int J Pharma Res Health Sci 6(2): 2342-2345.

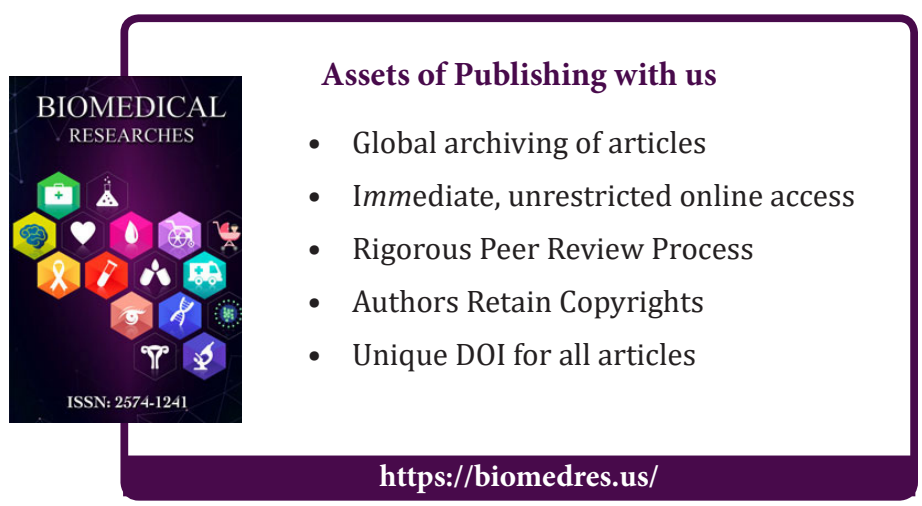

